\title{
Reflexiones acerca del término "Geolodáctica"
}

\author{
Reflections on the term "Geolodactics" \\ José Selés-Martínez \\ Departamento de Ciencias Geológicas, Facultad de Ciencias Exactas y Naturales, Universidad de Buenos Aires, Buenos Aires, Argentina. \\ E-MAIL: PEPE@GL.FCEN.UBA.AR
}

\begin{abstract}
The etymological correctness, the amplitude of meaning, and the need to introduce the term "geolodactics" into professional jargon are discussed and it is concluded that: a) it is incorrect in its construction, b) it is assigned a meaning considered excessive relative to the proposed scopes and c) it is unnecessary since there is the possibility of eliminating the ambiguity involved in the prefix "geo" (as it does not distinguish between its reference to geology or geography) using the expression "didactics of geology" in a way similar to how other specific didactics are referred to. It is also considered necessary to maintain the use of the expression "didactics of Earth Sciences" when referring to the teaching of knowledge from Geology, Geography and Atmospheric and Ocean Sciences, gathered under that name in the curricular contents of school education in many countries.
\end{abstract}

Resumen: Se discuten la corrección etimológica, la amplitud de significado y la necesidad de introducir en la jerga profesional la palabra "geolodáctica" y se concluye que: a) la misma es incorrecta en su construcción, b) se le asigna un significado considerado excesivo en cuanto a los alcances propuestos y c) la misma es innecesaria ya que existe la posibilidad de eliminar la ambigüedad que el prefijo "geo" presenta en cuanto no distingue entre su referencia a la geología o a la geografía utilizando la construcción "didáctica de la geología", en forma similar a como se hace referencia a otras didácticas específicas. Se considera también necesario mantener el uso de la expresión "didáctica de las Ciencias de la Tierra" en referencia a la enseñanza de los conocimientos provenientes de la Geología, la Geografía y las Ciencias de la Atmósfera y de los Océanos reunidos bajo esa denominación en los contenidos curriculares de la formación escolar en muchos países.
Citation/Citação: Sellés-Martínez, J. (2021). Reflexiones acerca del término "Geolodáctica". Terræ Didatica, 17(Publ. Contínua), 1-5, e021044. doi: 10.20396/td.v17i00.8665817.

Keywords:Didactics of Geology, Didactics of Earth Sciences, Etymology, Discussion.

Palabras clave: Didáctica de la Geología, Didáctica de las Ciencias de la Tierra, Etimología, Discusión.

\section{Manuscript/Manuscrito:}

Received/Recebido: 13/06/2021

Revised/Corrigido: 08/10/2021

Accepted/Aceito: 10/11/2021

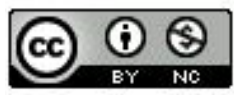

\section{Introducción}

Esta contribución se fundamenta en la necesidad de discutir la necesidad y la corrección etimológica del neologismo "geolodáctica" propuesto por Lacreu (2012, 2014, 2015, 2017a, b y c, 2019, 2020a y b) para denominar "el campo científico que investiga la transposición de la geología". De ninguna manera pretende abordar conceptos de la didáctica en sí misma ni contradecir las consideraciones sobre las particularidades e importancia que el estudio de los problemas vinculados al mejoramiento de la enseñanza y aprendizaje de la geología tiene, asunto en el cual se coincide con gran parte de los conceptos vertidos por su autor en las publicaciones mencionadas.

Se expondrán a continuación algunos conceptos introductorios y luego los argumentos que permiten demostrar que el neologismo propuesto no es correcto desde el punto de vista semántico (por lo que tampoco es claro en su significado), no es apropiado en tanto las incumbencias asignadas exceden largamente las implicancias de la didáctica y tampoco es necesario, ya que este campo del conocimiento puede ser claramente identificado como "didáctica de la geología".

\section{El problema de las denominaciones de una disciplina que aborda diferentes contenidos a diferentes escalas}

A medida que la didáctica de las ciencias pasa de las áreas más generales a las más específicas va incorporando diferentes contenidos y esto no debe ser perdido de vista en la discusión de la terminología, ya que esta - quiérase o no - estará ligada de un modo u otro a la escala a la que esté haciéndose referencia, es decir a los "contenidos" a enseñar y aprender a ese nivel de abordaje del tema.

En el siguiente esquema se presenta una primera aproximación a las escalas de abordaje de la 
enseñanza de las Ciencias, desde un primer nivel en el que se encuentran ciencias tan diversas como las naturales y sociales hasta los niveles específicos y especializados en áreas dentro de cada disciplina.

1. Ciencias en general: Naturales, Sociales, etc.

2. Ciencias naturales: Ciencias de la Tierra, Ciencias de la Vida, Ciencias del Espacio, Paleontología

3. Ciencias de la Tierra: Geología, Oceanografía, Ciencias Atmosféricas, Geofísica, Geoquímica.

4. Ciencias Geológicas: Disciplinas y especialidades geológicas.

$\mathrm{Al}$ abordar el problema a la escala de disciplinas geológicas, paradójicamente, estas se hacen menos específicas e incorporan contenidos conceptuales que provienen de otras ciencias y que, naturalmente, condicionan fuertemente su didáctica y deben ser resueltos con un abordaje integrador. Los procesos y fenómenos geológicos resultan de la activación de combinaciones de procesos físicos y químicos, por ejemplo, cuyo conocimiento y comprensión condiciona fuertemente su interpretación correcta y la construcción de conocimiento que sirva para la explicación y el pronóstico. Enseñar mineralogía desde el punto de vista de la composición y formación de los minerales es más parecido a enseñar química mientras que hacerlo desde el punto de vista de la estructura cristalina se asocia más a la enseñanza de algunos temas matemáticos, como las proyecciones geométricas y los teselados. Enseñar geología estructural, por su parte, requiere el apoyo de conceptos de mecánica y reología cuando se analizan los procesos y mecanismos de deformación, pero implica una fuerte componente de geometría proyectiva cuando se aborda la descripción y análisis de las formas y disposición espacial de las estructuras generadas por los procesos de deformación. La cartografía geológica asocia al conocimiento específico de la composición, geometría y relaciones entre los cuerpos de roca, conceptos vinculados a la geometría y al diseño gráfico, y su enseñanza tiene muy poco parecido con la de la hidrogeología, más vinculada a la hidrodinámica y a la química. Con estos ejemplos queremos señalar que, más allá de las características particulares asociadas a la didáctica de la geología, que muy bien señala Lacreu en las contribuciones citadas, debemos reconocer que, cuando se ingresa

1 La Paleontología vincula estrechamente a las Ciencias de la Tierra y las Ciencias de la Vida. en un determinado nivel de detalle, es evidente que la didáctica de la geología es una didáctica híbrida, que debe ocuparse de enseñar física, química, matemáticas y geología, estrechamente asociadas y en contextos muy particulares. Entendemos que esto no es exclusivo de la geología y se repite para cada una de las ciencias naturales, ya que las mismas en realidad representan un nivel de organización de la materia más complejo y menos aislado que en el caso de la física y la química, pero requieren de éstas para su correcta descripción e interpretación.

\section{La introducción de un neologismo}

El diccionario de la Real Academia Española define neologismo como un "vocablo, acepción o giro nuevo en una lengua" y, en general, se acepta que estos surgen frente a la aparición de algo antes inexistente o desconocido, a lo que es necesario referirse y que el neologismo reemplaza al extenso conjunto de palabras que sería necesario utilizar de no crearse el mismo. Naturalmente, todos los neologismos dejan de serlo una vez adoptados e incorporados al lenguaje habitual. Para una interesante reflexión acerca de los tecnicismos, neologismo y extranjerismos en el que se destaca la incidencia de las ciencias en la creación e incorporación de nuevos términos en el lenguaje puede consultarse RAE (sin fecha). En términos de su proponente (Lacreu, 2018):

El neologismo Geolodáctica es un acrónimo que resulta de la composición de dos términos que no solo intentan reflejar su filiación, sino que, tratándose de un único término, denota que el campo está configurado en base a la integración y no a la mera sumatoria de disciplinas" (Lacreu, 2012 p. 97). Así, se utilizó el prefijo "geolo" para adscribir a la disciplina geológica y el sufijo "dáctica" para la didáctica, prescindiendo de la raíz "geo" debido a que, al estar compartida por Geología y Geografía puede mantener a las confusiones ya señaladas (Lacreu, 2018).

Debe destacarse aquí que, desde el punto de vista lingüístico, "Geolo" no es un prefijo sino un fragmento de otra palabra, así como tampoco "dáctica" es un sufijo sino también un fragmento de otra palabra. De este modo podemos concluir que el neologismo propuesto no es un acrónimo en el sentido estricto del término ni está compuesto por un prefijo y un sufijo, sino que resulta de la unión de dos fragmentos más o menos capricho- 
sos de los términos geología y didáctica. Con un criterio similar podrían acuñarse términos equivalentes para todas las disciplinas ("geogradáctica", para la geografía, "biolodáctica" para la biología y así sucesivamente) lo cual no parece necesario ni conveniente.

Dado que una de las justificaciones que el autor invoca para la creación del neologismo es la ambivalencia del prefijo "geo" en cuanto puede referirse tanto a la geología como a la geografía, se realizará en el ítem siguiente una breve revisión del origen y uso de "geo" como prefijo.

\section{El prefijo geo y los términos derivados de él}

En el caso del desarrollo de los términos vinculados al estudio de nuestro planeta, ha sido uso tradicional utilizar neologismos que incorporaban el prefijo "geo". El origen del mismo se encuentra en el nombre de la deidad griega Gea (Gaya, Gaia, Gaea) cuyo equivalente en la mitología romana era la diosa Terra Mater o Tellus Mater. Gea representa a la deidad primigenia, que se encuentra en el origen de todo y es, por lo tanto, también la diosa madre (Fig. 1).

Algunos de los neologismos oportunamente creados con el prefijo "geo" constituyen hoy términos tan antiguos como geometría (medida de la Tierra) y geografía (descripción de la Tierra), otros son de edad intermedia como geología (tratado de la Tierra); otros más modernos como geodinámica o geofisica, por ejemplo, hacen referencia a la aplicación de una disciplina o estudio a la escala global, mientras otros como geotermia o geotecnia señalan la integración a la geología de conceptos físicos (térmicos o mecánicos, respectivamente) en función de una aplicación fuertemente orientada. Geoingeniería es un neologismo muy reciente vinculado al uso del conocimiento para modificar el clima a nivel global.

El hecho que la geometría ya no se vincula a la Tierra como planeta, sino que constituye una rama de las matemáticas, pone de manifiesto que el significado de los términos varía a lo largo del tiempo. Las ciencias que se dedican al estudio de la Tierra son actualmente la geografía (que ha derivado desde una fuerte componente inicial de geografía física hacia la geografía social), y la geología (que incorpora algunos aspectos de la geografía física).

En el tema que nos ocupa, la enseñanza de la geología y los problemas asociados a ella, se han acuñado ya términos y expresiones como "geodidáctica", "didáctica de la geología" o "didácti-

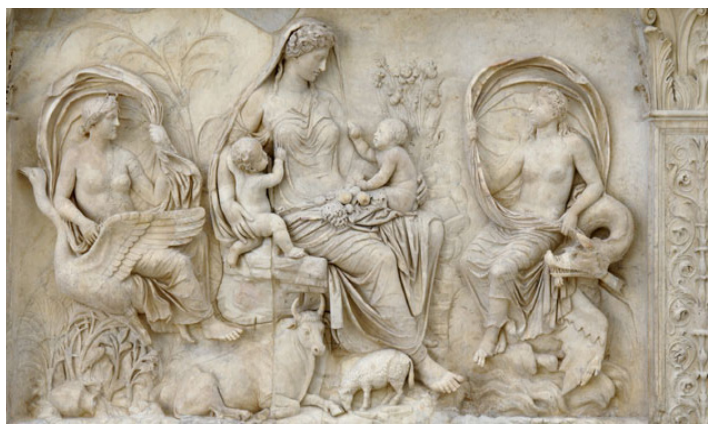

Figura 1. Relieve que representa a la diosa Tellus Mater. Museo del Ara Pacis, Roma (hacia el año 10 AC). Fuente: http://www.arapacis.it/es/didattica/ mitologia-storie-fantastiche-ed-immaginarie-0

ca de las ciencias de la Tierra". Dada la ambigüedad semántica de "geodidáctica", término en el que, como puntualiza Lacreu en las obras citadas, no es posible determinar si se refiere a la geología o a la geografía, sería recomendable discontinuar el uso del término y reemplazarlo por las expresiones "didáctica de la geología” y "didáctica de la geografía" según corresponda. Con respecto a la "didáctica de las ciencias de la Tierra", cuyo uso es desaconsejado por dicho autor, consideramos que el mismo es pertinente en tanto y en cuanto existe en los diseños curriculares una agrupación de contenidos que lleva ese nombre y que, en consecuencia debe enseñarse en forma conjunta e integrada, por lo que no bastaría unir fragmentos de las didácticas de la geología, de las ciencias de la atmósfera y de la de las ciencias del océano (o incluso de la didáctica de las ciencias del espacio, en los casos en que el área incluya algunos conceptos de astronomía) para abordar el problema. Es oportuno señalar aquí que son varias las instituciones que nuclean a docentes de geología que llevan la denominación "Ciencias de la Tierra", entre las que pueden mencionarse la Earth Science Teachers Association (ESTA) de Inglaterra, la National Association of Geosciences Teachers (NAGT) de los Estados Unidos de América y la Asociación Española para la Enseñanza de las Ciencias de la Tierra (AEPECT). La primera de ellas, si bien utiliza el término en singular, en su lema se señala que "promueve la enseñanza de la Ciencia de la Tierra a través de la Geología, la Geografía y las Ciencias" y en su publicación periódica utiliza el término en plural (Teaching Earth Sciences). Tanto ESTA como NAGT, en torno a los años `90, cambiaron su denominación original -que empleaba el término "Geología"- por la expresión "Ciencias de la Tierra" probablemente por considerarlo más abarcador y útil a la hora de referirse a la enseñanza

\begin{tabular}{c|c|c|c|c|c|}
\hline (C) Terrae Didat. & Campinas, SP & v.17 & $1-5$ & $\mathrm{e} 021044$ & 2021 \\
\hline
\end{tabular}


a nivel pre-universitario. La AEPECT, fue fundada en 1991 incorporando esa expresión a su nombre y al de su revista, pero conservó el término "Geología" en la denominación de los simposios que organiza bianualmente, ya que estos se iniciaron en 1980 y se consideró importante mantener el nombre tradicional.

\section{Una cierta desproporción entre el neologismo y el significado que se le asigna}

En Lacreu (2019) el autor define la geolodáctica como "el ámbito en el que los geolodactas investigan sobre los procesos de alfabetización geológica en todas sus dimensiones (didáctica, política, cultural, laboral, propedéutica)", aclarando que la definición es "provisoria" (véase también la figura 2, tomada de Lacreu (2020). Se considera que la pretensión de extender tan ampliamente el campo de investigación de quienes se ocupan de la enseñanza de una ciencia es desproporcionada. No se desconoce la importancia de la inclusión, en el proceso de enseñanza, de aspectos políticos, culturales, laborales, etc., ya que estos, además de su interés específico, pueden servir también como elementos motivadores del aprendizaje y como disparadores de proyectos de investigación escolar y ser motivo para la reflexión, pero debe tenerse en cuenta que estos temas ya están presentes (si bien en una proporción más reducida que la que se desprendería de la propuesta por Lacreu) en todas las didácticas modernas en tanto y en cuanto estas incorporan la consideración del contexto en el que tiene lugar el proceso de trasmisión de saberes. Naturalmente cómo se enseña no puede separarse de qué se enseña y para qué, pero se considera que no debería ser la didáctica el campo donde estas cuestiones se discutan.

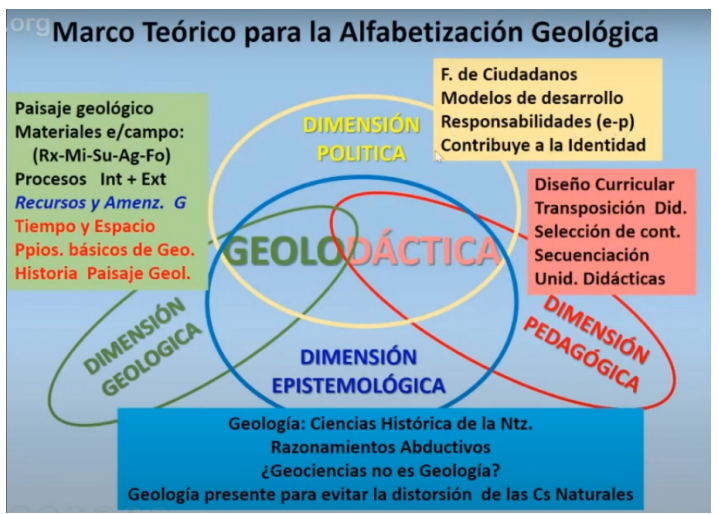

Figura 2. Marco teórico para la Alfabetización Geológica captura de pantalla tomada de Lacreu (2020b)

\section{Conclusiones}

Si bien se coincide en términos generales acerca de los problemas asociados a la enseñanza de la geología, que acertadamente señala Lacreu en los trabajos citados, se señalan como conclusiones de la presente contribución:

a. Se considera que no es prudente incorporar a la jerga el término "geolodáctica" por las razones mencionadas, dado que el mismo es incorrecto en su construcción, ambiguo en su significado y sobredimensionado en las implicancias que se le asignan.

b. Se defiende la continuidad del uso de la expresión "didáctica de la geología" para referirse a la enseñanza de esta disciplina, ya que la misma es suficientemente clara y análoga a otras expresiones como didáctica de la biología o didáctica de las matemáticas, por ejemplo.

c. Se defiende la continuidad del uso de la expresión "didáctica de las ciencias de la Tierra" cuando se hace referencia a la enseñanza de los contenidos agrupados en esta asignatura curricular o agrupamiento de contenidos disciplinares.

d. Se propone el abandono del uso de la palabra "geodidáctica" por la ambigüiedad del significado del prefijo geo, que no distingue entre su aplicación a la geografía o a la geología.

\section{Referencias}

Lacreu, H. L. (2012). Raíces políticas del analfabetismo geológico. XVII Simposio sobre Enseñanza de la Geología. Comunicaciones: 91-99, Huelva, España.

Lacreu, H. L. (2014). Aciertos, distorsiones y falacias en la enseñanza de las ciencias naturales de la educación secundaria obligatoria de Argentina. Terree Didatica, 10(3), 217-226. Campinas, Brasil. doi: 10.20396/td.v10i3.8637323.

Lacreu, H. L. (2015). Geociencias para la formación ciudadana. XIV Congreso Geológico Chileno, Actas:469-472. La Serena, Chile. URL: https:// www.researchgate.net/publication/317721970 Geociencias_para_la_formacion_ciudadana. Acceso 11.04.2021

Lacreu, H. L. (2017a). Desafíos de la Alfabetización Geológica. XX Congreso Geológico Argentino, Actas: 20-24, Tucumán, Argentina. URL: https:/www. researchgate.net/publication/328792664_Desafios de la Alfabetizacion Geologica. Acceso 11.04.2021.

Lacreu, H. L. (2017b). El paisaje geológico en la enseñanza de las Geociencias: ¿̇es un recurso didáctico, es un objeto de estudio o ambas cosas a vez? Enseñanza de las Ciencias de la Tierra, 25(3), 310-318. URL: https://www.raco.cat/index.php/ECT/arti- 
cle/view/330136. Acceso 25.05.2021.

Lacreu, H. L. (2017c). The Social Sense of Geological Literacy, In: Peppoloni, S., Di Capua, G., Bobrowsky, P. T., \& Cronin, V. (Eds.). Geoethics at the heart of all geosciences. Annals of Geophysics, 60(Fast Track 7). doi: $10.4401 / \mathrm{ag}-7558$.

Lacreu, H. L. (2019). Geolodáctica, desafíos para renovar la enseñanza de la Geología. Terra Didatica, 15(Publ. Contínua), 1-11, e019017. doi: 10.20396/ td.v15i0.8654666.

Lacreu, H. L. (2020a). Geolodáctica para el estudio del paisaje natal. Alambique: Didáctica de las ciencias ex- perimentales, 102, 47-54.

Lacreu, H. L. (2020b). Introducción a la Clínica Geolodáctica. San Luis, Argentina. URL: https://www. youtube.com/watch?v=L-702Tj6Kn4. Acceso 11.04.2021.

RAE (sin fecha). Tecnicismos, neologismos y extranjerismos en el español. Real Academia Española de la Lengua https://www.rae.es/sites/default/files/TecnicismosNeologismosExtranjerismos.pdf. Acceso 31.10.2021.

Wikipedia (2021). Neologismo. URL: https://es.wikipedia.org/wiki/Neologismo. Acceso 11.04.2021. 